der“ als die Zeitung, „Kassetten-Fernsehen“ generell „avancierter“ als das Fernsehen? Vielmehr: Werden sie im zeitgenössischen Diskurs wirklich so aufgefasst?

Auch manche Detailaussagen scheinen problematisch - und nicht wenige kryptisch bis schlicht unverständlich. So, wenn Christian Kassung über die Technik der Lithographie aussagt: „Der Stein rauscht mit einer Wahrscheinlichkeit von zwei Dritteln, gleichzeitig aber lässt sich auf ihm völlig störungsfrei arbeiten “ (S. 89), oder wenn er darstellt, Carl Maria von Weber habe „halt nichts aus der Lithographie machen können, weil er später ein berühmter Komponist geworden ist. Ein derartiger Rekurrenzeffekt zeigt sehr eindrücklich, dass sich Mediengeschichte im Archiv seiner Diskurse immer schon aufgeschrieben hat" (S. 80 f.); so auch, wenn Leander Scholz über den Buchdruck ausführt: „Auf welche Weise ein Medium jeweils diskursiviert wird, hängt mithin entscheidend davon ab, welches Interface ihm als menschliches Gesicht und damit als seine Verständlichkeit zugeordnet werden kann“ (S. 14). Zumindest scheint hier und an vielen anderen Stellen Sprache weit mehr als Verschleierungs- denn als Verständigungsmittel eingesetzt.

Was weiter gänzlich fehlt, sind resümierende Zusammenfassungen am Ende jedes Textes und ein Fazit, dass zum Abschluss des Bandes noch einmal die verschiedenen Aspekte bündeln könnte. Die sechs genannten „Problemfelder“ werden jeweils abgehandelt - dann ist unvermittelt Schluss.

Insgesamt bietet das Buch manche interessante Beobachtungen, einige Ansätze zur Diskussion über den Diskurs über Medien, eine Reihe schöner Illustrationen, auch einen innovativen Zugang, den man als einen Aspekt unter anderen heranziehen kann, wenn es darum geht, Mediengeschichte umfassender zu schreiben. Eine konzise „Einführung in die Geschichte der Medien" ist das aber nicht. Und der „Gebrauchswert" etwa in der universitären Lehre, ein Anliegen ja gerade der UTB-Veröffentlichungen, ist allenfalls gering.

Markus Behmer

\section{Christoph Kaletka}

\section{Die Zukunft politischer Internetforen}

Eine Delphi-Studie

Münster: LIT, 2003. - 244 S. [Anh. 7 S.]

(Reihe Medienzukunft heute; 9)

ISBN 3-8258-7117-7

Das Internet und dessen vermutete Folgen für unsere Gesellschaft sind seit Jahren zentrale Forschungsgegenstände nicht nur der Kommunikationswissenschaft. Auch Christoph Kaletka interessiert sich für diesen Gegenstand und nimmt dabei mit politischen Internetforen die Schnittstelle von Politik und Mediensystem in den Blick. Auch wenn die Relevanz des Gegenstands in der Einleitung mit z. T. bereits in der Vergangenheit gescheiterten Projekten politischer Onlineforen begründet wird, geht der Blick des Forschers methodisch in die Zukunft: Mit Hilfe der Delphi-Methode soll aufgeklärt werden, welche Entwicklungen im Bereich politischer Onlineforen vor einem Zeithorizont von etwa 10 Jahren von Experten für wahrscheinlich gehalten werden. Ziel des Vorgehens ist es insbesondere, „Orientierung für die Anbieter und Nutzer politischer Foren“ (12) zu bieten, wofür kommunikationswissenschaftliche Modelle und Methoden herangezogen werden.

Der Gegenstand „politische Internetforen“ wird vom Autor in der Einleitung etwas versteckt definiert und eingegrenzt als Informations- und Diskussionsangebote im World Wide Web (WWW), die von verschiedensten Akteuren betrieben werden können. Der Autor verzichtet bewusst auf eine „allgemein gültige Definition " (50), da das weitere induktive Vorgehen dadurch behindert würde. Eine operationale Definition wird für das Vorgehen dennoch benötigt, sie findet sich in dem im Anhang abgedruckten Fragebogen, den die Experten zur Beantwortung erhalten hatten: „WWW-basierte Sites, die Interessierten die Möglichkeit zum Meinungsaustausch zu politischen Themen bieten" (246).

In zwei Theoriekapiteln werden anschließend die beiden vom Untersuchungsgegenstand berührten Forschungsfelder „Politik“ und „Kommunikation“ behandelt. Zuerst wird die Bedeutung von Partizipation und Kommunikation im demokratischen politischen Prozess erläutert. Insbesondere werden hier diejenigen Aspekte von Politik herausgearbeitet, für 
die der Untersuchungsgegenstand der Internetforen prinzipiell von Bedeutung sein kann, insbesondere politische Partizipation, die Rolle von Information und Kommunikation im politischen Prozess und die sich bereits herausbildenden Formen verschiedener Internet-basierter politischer Aktionsformen. Kapitel 3, mit „kommunikationswissenschaftlichen Perspektiven" überschrieben, thematisiert zuerst die Entwicklungsgeschichte des Internets, verschiedene Erscheinungsformen der OnlineKommunikation sowie schließlich etwas ausführlicher die Frage, welche Veränderungen das Konzept der „Öffentlichkeit“ im Internet erfährt.

Unangenehm fällt leider in beiden Theoriekapiteln auf, dass die zentralen Begriffe relativ oberflächlich und, wenn überhaupt, meist nur auf Basis von Sekundärliteratur definiert und abgehandelt werden. Ob hierbei die wesentlichen relevanten Aspekte von "Partizipation“, „Direkter Demokratie“, „Cyberdemocracy“ etc. erfasst und in die theoretische Basis der Untersuchung eingearbeitet werden konnten, ist zumindest fraglich. Natürlich ist es durchaus legitim, sich für eine bestimmte Definition und Systematisierung eines Konzepts zu entscheiden, wie dies hier beispielsweise für die zweidimensionale Definition von Partizipation anhand der Kriterien „Unmittelbarkeit“ und „Verfaßtheit“ nach von Alemann (1975) geschehen ist; eine Begründung dieser Wahl, etwa durch Diskussion mehrerer Definitionsvorschläge oder durch Verweis auf weitere Literatur, die diese Wahl unterstützt, würde aber der intersubjektiven Nachvollziehbarkeit der Argumentation nützen. Dies gilt in gleichem Maße für die gelegentlich ohne Beleg aufgestellten Behauptungen, die dem Leser ein zu großes Maß an Vertrauen in den Autor abfordern. Unbelegte Aussagen, wie „die Mehrzahl der Autoren“ hätte bestimmte Erklärungen für Phänomene wie Politikverdrossenheit (26) oder das Radio sei ursprünglich zum Zweck der interpersonalen Kommunikation entwickelt worden (89), sind eher geeignet, Missverständnisse oder beliebte Mythen weiter zu verselbstständigen als wissenschaftlichen Erkenntnisgewinn zu befördern.

In Kapitel 4 wird schließlich die Theorie übergeleitet in ein vierdimensionales Untersuchungsmodell, das die empirische Erhebung strukturieren soll. In den vier Abschnitten „Medien“, „Politik“, „Nutzung“ (evtl. treffen- der mit „Funktion“ betitelt) und „Technik“ wird jeweils eine Reihe von Thesen formuliert, zu denen sich die befragten Experten äußern sollen. Hier werden die Elemente, die im Theorieteil noch eher assoziativ verknüpft wurden, systematisch und nachvollziehbar auf einander bezogen und ergeben ein in sich schlüssiges Modell. Wenn diese Dimensionen, deren Herkunft zu Beginn dieses Abschnitts nicht ganz klar wird, bereits in der Struktur der beiden Theoriekapitel angelegt gewesen wären, wäre dieser Teil in seiner Struktur wohl etwas besser durchschaubar.

Im empirischen Teil ab Kapitel 5 liegt die Stärke des Buches. Das methodische Vorgehen ist unter Verweis auf zentrale Methodentexte nachvollziehbar begründet und kann gut in die - wie der Autor zeigt - relative kurze Geschichte der Delphi-Forschung eingeordnet werden. Auch das konkrete empirische Vorgehen ist gut nachvollziehbar. Sowohl die einzelnen Schritte des Prozesses als auch zentrale Qualitätskriterien sowie das Erhebungsinstrument sind dokumentiert und können zur Bewertung der Ergebnisse herangezogen werden. Der Autor wertet die einzelnen Abschnitte der Expertenbefragung einzeln nacheinander aus und extrahiert aus jedem eine generelle Prognose, etwa: „Politiker werden ... verstärkt auf Kommunikation in Internetforen reagieren: Das Wirkungspotenzial von Politikforen steigt" (205). Generell stellt er fest, dass die Experten den Foren eine durchaus lebhafte $\mathrm{Zu}$ kunft bescheinigen. Basis dieser Erkenntnisse sind die Antworten der Experten in zwei Befragungswellen, bei denen insbesondere die Antworten nach der zweiten Welle, nachdem die Experten die Ergebnisse der ersten Welle zur Kenntnis genommen haben, die Indikatoren für deren Einschätzungen bilden. Bei der Interpretation der Ergebnisse muss man jedoch darauf achten, dass es sich eben um Vermutungen - wenn auch um durchaus begründete, auf Erfahrung und hoher Fachkompetenz beruhend - handelt. Eine Aussage wie „Nutzer wollen Foren auch als Kontaktbörse nutzen“ (218) ist eben keine Aussage über die Nutzer, sondern eine Aussage über die Vermutungen von Experten über Nutzer.

Auch wenn solche mit Hilfe der Delphi-Methode gewonnenen Erkenntnisse grundsätzlich wenig zur Gewinnung sicherer Erkenntnisse über den Gegenstand der Internetforen beitragen können, so sind sie mit Sicherheit erstens 
wertvoll für Akteure, deren heutiges Handeln von der Abschätzung zukünftiger Entwicklungen abhängt, also insbesondere politische und wirtschaftliche Akteure. Und da zweitens ein ganz wesentliches Manko der verwendeten Methodik darin liegt, wie Häder (1996: 4) feststellt, dass ihre Prognosequalität empirisch noch unzureichend überprüft ist, weist der $\mathrm{Au}-$ tor am Ende zu Recht auf die zukünftig mögliche und auch nötige empirische Prüfung der mit dieser Untersuchung aufgestellten Hypothesen hin, die einen weiteren Erkenntnisgewinn fördern kann.

\section{Martin Emmer}

\section{Literatur:}

Alemann, Ulrich von (1975): Partizipation Demokratisierung - Mitbestimmung. Problemstand und Literatur in Politik, Wirtschaft, Bildung und Wissenschaft. Eine Einführung. Opladen: Westdeutscher Verlag.

Häder, Michael (1996): Zur Evaluation der Delphi-Technik. Eine Ergebnisübersicht. ZUMA-Arbeitsbericht 96/02.

\section{Michael Beuthner / Joachim Buttler / Sandra Fröhlich / Irene Neverla / Stephan A. Weichert (Hrsg.)}

\section{Bilder des Terrors - Terror der Bilder?}

Krisenberichterstattung am und nach dem 11. September

Köln: Herbert von Halem Verlag, 2003. 377 S.

\section{ISBN 3931606562}

Vielen Herausgebern und Verfassern (populär-) wissenschaftlicher Literatur über den 11. September 2001 ist - teils zu Recht - der Vorwurf gemacht worden, als Akademiker in forschungsstrategischer Eile in die journalistische Aktualitätsfalle getreten zu sein. Tatsächlich haben in unmittelbarer Folge der Terroranschläge islamischer Extremisten in den USA 2001 zahlreiche verlegerische Schnellschüsse von oft zweifelhafter Qualität den Buchmarkt, sprich: die Frankfurter Buchmesse getroffen. Der von Michael Beuthner et al. herausgegebene Band dagegen zählt zu den raren erkenntnisinteressanten Ausnahmen. Die Dokumentation einer gleichnamigen Tagung im November 2002 in Hamburg überzeugt dank ihres inter- disziplinären und praxeologischen Ansatzes: Journalisten und Wissenschaftler unterschiedlicher Disziplinen wie Kommunikations- und Medienwissenschaft, Kunstgeschichte, Kulturwissenschaft, Politologie, Soziologie, Sozialphilosophie, Psychologie und Kriminologie hinterfragen in insgesamt 18 Fachbeiträgen und einer dokumentierten Podiumsdiskussion die Ambivalenz der Krisenberichterstattung am und nach dem 11. September als Medienereignis. Im Forschungsfokus stehen einerseits die aktualitätsfetischistische globale EchtzeitÜbertragung von Terror-Bildern der einstürzenden Zwillingstürme des World Trade Center und andererseits die Berichterstattungsredundanz in Form dramatisierter EndlosWiederholungsschleifen, die zu einem BilderTerror führten.

Die Herausgeber gehen von der etwas verfrüht formulierten Prämisse aus, der 11. September sei „eine Zäsur - politisch, kulturell, medienhistorisch" (S. 8) gewesen. In der noch jungen post 9/11-Medienära sind zwar oberflächliche Veränderungen in der Visualisierung und Kontextualisierung der Krisenberichterstattung zu beobachten. Fraglich ist aber, ob und inwieweit sich mittelfristig die redaktionellen und organisatorischen Tiefenstrukturen bzw. Infrastrukturen ändern. Denn weiterhin mangelt es den meisten Nachrichtenmedien an Nachhaltigkeit in der Krisenberichterstattung genauso wie an institutionalisierten Koordinations- und Clearingstellen für den Krisenfall. Das in ihrem Essay engagiert geführte und konstruktive Plädoyer von Irene Neverla für eine „Qualitätsoffensive auf der Grundlage der Ressortkompetenz ,Krisenjournalismus “ (S. 161), die auch durch „die Bereitstellung von Aufklärungssatelliten für journalistische Zwecke“ (S. 162) gestützt werden solle, ist leider noch weit von einer Umsetzung in die Praxis entfernt.

Plausibel fordern Michael Beuthner und Stephan Alexander Weichert in ihrer Einführung „das künftige akademische Projekt einer interdisziplinären Bildkritik“ (S. 13) als Ergänzung zur alt bewährten Textkritik. Folgerichtig reflektieren gleich drei Beiträge im ersten Buchteil „Von den Terrorbildern zur Medienerzählung" die Ikonologie des 11. September, dessen televisuelles Terror-Zeugnis Einzug ins menschliche Kollektivgedächtnis gefunden hat. Joachim Buttler arbeitet in seinem Beitrag überzeugend die Symbolgeschichte der Zer- 